\title{
Endogenous preference formation on macroeconomic issues: the role of individuality and social conformity
}

\author{
Guido Baldi
}

Received: 13 July 2013/Accepted: 20 January 2014/Published online: 8 March 2014

(C) Springer-Verlag Berlin Heidelberg 2014

\begin{abstract}
Macroeconomic events often require individuals and policy-makers to make decisions that they are not accustomed to making. For example, a sovereign debt crisis makes it necessary to either default on government debt, increase taxes, cut public spending or to impose a mixture of these measures. I argue that decisions on such matters are not derived from deep preferences; they require reflections and judgement under uncertainty. Past experiences and the interaction with other individuals are likely to influence the salience of preferences in the situations of decision making. Using a simple model, I illustrate how the salience of preferences changes with different degrees of individuality and conformity. Individuality is associated with the importance of private habits, while conformity is related to the perceived dissonance between initial intuitions and social opinions. The results obtained from simple simulation exercises stress that a high degree of conformity or a low degree of individuality may lead to overreactions when social opinion makers err for a short period of time. At the same time, a low degree of conformity or a high degree of individuality or leads to delayed adjustments to new circumstances.
\end{abstract}

Keywords Preference formation · Rationality $\cdot$ Habits

JEL classification $\quad \mathrm{B} 41 \cdot \mathrm{B} 52 \cdot \mathrm{E} 02$

\footnotetext{
G. Baldi

Department of Economics, University of Bern,

Schanzeneckstr. 1, 3000 Bern, Switzerland

G. Baldi (凶)

Department of Macroeconomics and Financial Markets,

German Institute for Economic Research (DIW), Mohrenstr. 58, 10117 Berlin, Germany

e-mail: guido.a.baldi@gmail.com
} 


\section{Introduction}

Decisions on macroeconomic issues are often characterized by a high level of abstractness, uniqueness and uncertainty. For instance, if a sovereign debt crisis occurs, should the government default on its debt, or implement spending cuts and tax increases? Or, should a central bank adopt an inflation target? As I will argue in this paper, individuals cannot be expected to have so-called "deep" preferences on such issues. I propose a simple way to model the formation of preferences as the result of an interaction between what I call private motivating intuitions (or initial convictions) and social opinions. Preference formation is rational in the sense that individuals reflect on their preferences and do not act thoughtlessly. This is modeled as a process in which the salience of preferences is influenced by the degree of individualism and conformity. In this way, the preference-formation process provides reasons for preferences and preference modifications. As I refer to a minimal definition of rationality, full consistency or transversality are not necessarily attributes of rational behavior. While the macroeconomic debate on rationality mainly centers on the formation of expectations, I focus on the role of rationality in the formation of preferences.

Using a simple model, I illustrate how the salience of preferences changes with different degrees of individuality and conformity. Individuality is associated with the persistence of private motivating intuitions or convictions and conformity is related to the perceived dissonance between private intuitions and social opinions. The results of simple simulation exercises where experts or policymakers influence social opinions stress the importance of individuality in determining the salience of preferences. A high degree of individuality or a low degree of conformity prevents individuals from overreacting. A low degree of individuality and a high degree of conformity, however, lead to overreactions in cases where social opinion makers (for example experts or policy-makers) err for a short period of time. But high individuality and low conformity may also have drawbacks: if the degree of conformity is low or the degree of individuality is high, delayed adjustments may be observed.

As mentioned above, I assume that an individual's action-inducing preferences over possible worlds, states or outcomes depend on two factors: the intuitions that motivate the individual and social opinions that implicitly attach different weights to the motivating intuitions. Social opinions are influenced by "opinion-makers" such as experts, policy-makers or journalists. In the approach taken in this paper, social opinions are not chosen strategically and cannot be attributed to persons. An active interaction between individuals and social opinions is therefore not modeled in this paper. Both private intuitions and social opinions are essential for individual behavior. Changes in social opinions can cause changes in private habits by giving rise to new perceptions and dispositions within individuals. In this way, changes in social opinions produce downward effects. When deriving preferences, individuals partly interiorize social opinions. These arguments are related to the concept of procedural rationality proposed by Simon (1976), according to which the choice at a particular point in time is context-specific. This involves a rejection of the idea that human nature, or at least the motives, goals and preferences of individuals are 
entirely psychologically given. However, as it is shown in this paper, this does not involve a rejection of the importance of individuality.

In psychological terms, one could argue that private motivating intuitions are produced by the "fast system", while the action-inducing preferences are determined by the "slow system". In this way, the motivating intuitions together with the social opinions determine the salience of preferences. It has been shown that the past influences the current salience of preferences. This brings us to the notion of habits. Habits are propensities for responding in a specific way to a particular class of situations (see, for instance, Rutherford 1994). Repetition is an important feature of habits. Habits, however, are much more complex than mere repetitions and involve the imitation of others, which makes habits closely related to social institutions. According to Hodgson (2004), “...the reason why we have evolved the capacity to form habits is to deal with the uncertainty, complexity and variability of circumstances...". This stresses the importance of studying habits in the context of macroeconomics and, in particular, rare macroeconomic events that are often associated with uncertainty, complexity and uniqueness. The understanding of the notion "habit" in this paper as being related to preferences differs from the understanding in parts of the macroeconomic literature, where habits are formed over economic variables such as consumption and may yield correlated behavior or correlated choice over time. ${ }^{1}$

By acknowledging the existence of habits, I argue that macroeconomics should take into account that preferences of individuals can be endogenous, in particular over the medium- and long-term. In this paper, preferences of individuals are the result of an interaction between private motivating intuitions (influenced by private habits) and social habits or, as I call them, social opinions. Social opinions act as a weighting function that individuals use to attach different weights to their intuitions. By emphasizing the importance of private intuitions and social opinions, the model of this paper does not strictly adhere to methodological individualism. But, at the same time, individuals are not exclusively seen as purely social beings. Private motivating intuitions are influenced by private habits and are associated with individuality. Conformity is characterized by collective behavior. Conformity implies that the social status is sufficiently important for an individual. Individuals depart from their heterogenous intuitions or convictions to partly conform to a single, more homogeneous way of behavior.

The arguments made in this paper are inspired by various sources. The importance of habits and endogenous preferences was stressed long ago by the proponents of the early institutionalist movement in the 1920s and 1930s (see Rutherford 1994 or Hodgson 2004 for useful overviews). In addition, this paper draws on the literature on endogenous preferences in microeconomics (see, for example, Rabin 1994, 2013; Bowles 1998, 2004). Conformity has been analyzed in an economic model, for instance, by Bernheim (1994). Interpreting social habits or opinions as a weighting function for private motivating intuitions has certain relations to the arguments in Dietrich and List (2013), and the discussion in the philosophy and economics literature on reasons for preferences. Recently, Hoff and

\footnotetext{
$\overline{1}$ See, for example, Ravn et al. (2006).
} 
Stiglitz (2010) introduced the notion of "social constructs", which is very similar to the notion of social habit used by others and the notion of social opinions in this paper. They argue that social constructs provide the cognitive frames that individuals use to shape their perception. Similar to my arguments, Rodrik (2014) uses the notion of "ideas" to describe how policy decisions are derived. My paper is also loosely related to the literature on social capital (see, for instance, Knack and Keefer 1997), although I do not interpret social opinions as being capital stock. As noted by Rabin (2013) and Harstad and Selten (2013), trying to improve the psychological realism of economics does not necessarily imply that one has to entirely reject the concept of optimization. Instead, incorporating the impact of habits or the desire for conformity can be done within the existing modeling framework.

The rest of this paper is organized as follows. Section 2 illustrates a simple modelling approach of endogenous preference formation that takes into account the private and social dimension of preference formation. Section 3 presents the results of simple simulation exercises. Finally, Sect. 4 contains the conclusion.

\section{Modeling individuality and social conformity}

This section describes a modeling approach for the formation of preferences that incorporates private intuitions and social opinions. I propose a way to link the private and social sphere by the desire of individuals to act in conformity with their social environment. Individuality is modeled as the importance for an individual not to deviate from his private habits or private convictions. An individual first develops motivating intuitions that start the process of finding underlying reasons for actioninducing preferences. The set of motivating intuitions that is relevant for an individual at a given point in time is denoted by $I$. These motivating intuitions are relevant if the individual perceives to be in a state in which these intuitions are relevant. $^{2} I$ is a subset of an infinite set of possible intuitions $M$, which includes all the intuitions that can become motivating for an individual.

The motivating intuitions provide an individual with an initial salience of a preference. In a public debt crisis, for instance, an individual may develop intuitions over measures to cope with the crisis. Such measures may consist of tax increases, government spending reductions or sovereign default. If an individual remembers having experienced a similar situation before, he may, for instance, intuitively favor tax increases over spending cuts and this may in turn be preferred to default. If the individual does not remember having experienced a similar situation before, he will be intuitively indifferent toward these alternatives. Consider an example where a country is in a serious public debt crisis that results in high deficits and public debt that needs to be cut. I assume that in the state of a crisis, the following private intuitions may become salient:

$(S)$ The country should reduce spending, because this solves the crisis.

\footnotetext{
2 This perspective is similar to the approach in Dietrich and List (2013), although the methodology used in their paper is different from the one in this paper.
} 
(T) The country should increase taxes, because this solves the crisis.

(D) The country should default on its debt, because this solves the crisis.

For instance, having the intuition $(S)$ implies that an individual consciously or unconsciously has a model in mind according to which spending cuts represent the best way to deal with a debt crisis. Instead, having the intuition $I=(S, T)$ means that an individual's intuition tells him that the country should both increase taxes and reduce spending in order to solve the public debt crisis. Other potential intuitions of an individual may be, for example, $I=(S, T)$ or $I=(D)$. Clearly, two individuals can well have different intuitions allowing for a nuanced perspective on disagreements between two individuals. Intuitions are formed by what psychologists may call the automatic or fast system.

With time, an individual starts to reflect on his intuitions. When reflecting on his reasons, an individual is influenced by private habits and the degree of dissonance between private intuitions and social opinions. As Elster (2009) said, “....a reason demands the reason for that reason...". Private habits and social opinions help an individual reflect on his intuitions and provide the reasons for the reasons. Individuals develop private habits in the salience of their intuitions, which implies that they have a desire to avoid variations in their preferences. The social opinions provide the thinking framework while an individual learns from society in the course of experience. They impact on the individual via his desire to reduce the perceived degree of social dissonance. Following this reasoning, an individual $i$ maximizes the following function in trying to extract the maximum possible salience over possible alternatives:

$$
\max _{Z_{t}^{p}} Z_{t}^{s}(i) Z_{t}^{p}(i)-\frac{\kappa}{2}\left(Z_{t}^{p}(i)-\beta Z_{t-1}^{p}(i)\right)^{2}-\frac{\mu}{2}\left(Z_{t}^{p}(i)-Z_{t}^{s}(i)\right)^{2}
$$

$Z_{t}^{p}(i)$ is the private salience and $Z_{t}^{s}(i)$ is the social opinion. In the following, I am going to denote $Z_{t}^{p}(i)$ and $Z_{t}^{s}(i)$ by $Z_{t}^{p}$ and $Z_{t}^{s}$ for ease of discussion. The desire of an individual to act in conformity with social opinions is captured by $\mu$ that weights the “conformity term" $\left(Z_{t}^{p}-Z_{t}^{s}\right)^{2}$. In addition, social opinions also act as weights of importance for the individual in the term $Z_{t}^{s} Z_{t}^{p}$. $\kappa$ determines how much an individual dislikes to deviate over time from his private habits or convictions. $\beta$ stands for the degree of forgetting past convictions. It can be considered as a memory parameter and determines the persistence of private habits. Therefore, we call it the parameter that captures the degree of individuality. Using the squared disutility terms $\frac{\kappa}{2}\left(Z_{t}^{p}-\beta Z_{t-1}^{p}\right)^{2}$ and $\frac{\mu}{2}\left(Z_{t}^{p}-Z_{t}^{s}\right)^{2}$ implies that the sign of the difference between the two terms inside the parenthesis does not impact on the size of the cost. The assumption of quadratic costs for deviations from private habits and social dissonance and the implied linearity of the first-order condition keep the simple model tractable. Maximizing (1) with respect to $Z_{t}^{p}$ gives the first-order condition:

$$
Z_{t}^{s}-\mu\left(Z_{t}^{p}-Z_{t}^{s}\right)-\kappa\left(Z_{t}^{p}-\beta Z_{t-1}^{p}\right)=0
$$

This can be rearranged to get an expression for the evolution of the salience of the preference $Z_{t}^{p}$ : 


$$
Z_{t}^{p}=\frac{\kappa \beta \cdot Z_{t-1}^{p}+(1+\mu) \cdot Z_{t}^{s}}{\mu+\kappa}
$$

This difference equation in $Z_{t}^{p}$ implies that, in a long-run stationary equilibrium, the private salience equals the salience of the social opinion (that is $\bar{Z}^{p}=\bar{Z}^{s}$ ), if $\left(\frac{\mu+\kappa-\kappa \beta}{\mu+\kappa}\right)=\frac{(1+\mu)}{\mu+\kappa}$, or, simplified: $\kappa(1-\beta)=1$. For the simulation exercises of the

Table 1 Initial values of the salience of private intuitions

\begin{tabular}{ll}
\hline Individual & Salience of private intuitions \\
\hline 1 & 0.1 \\
2 & 0.2 \\
3 & 0.3 \\
4 & 0.35 \\
5 & 0.5 \\
6 & 0.6 \\
7 & 0.7 \\
8 & 0.8 \\
9 & 0.9 \\
10 & 0.95 \\
\hline
\end{tabular}

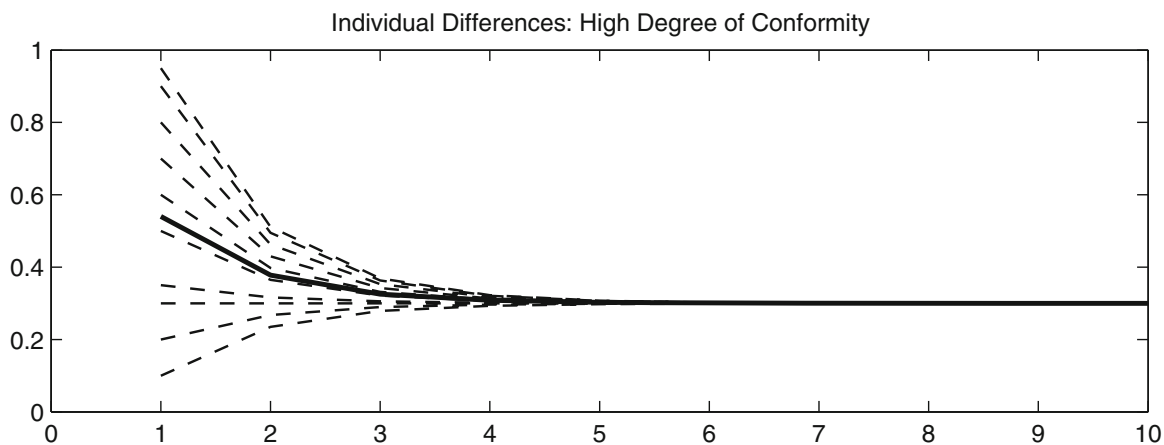

Individual Differences: Low Degree of Conformity

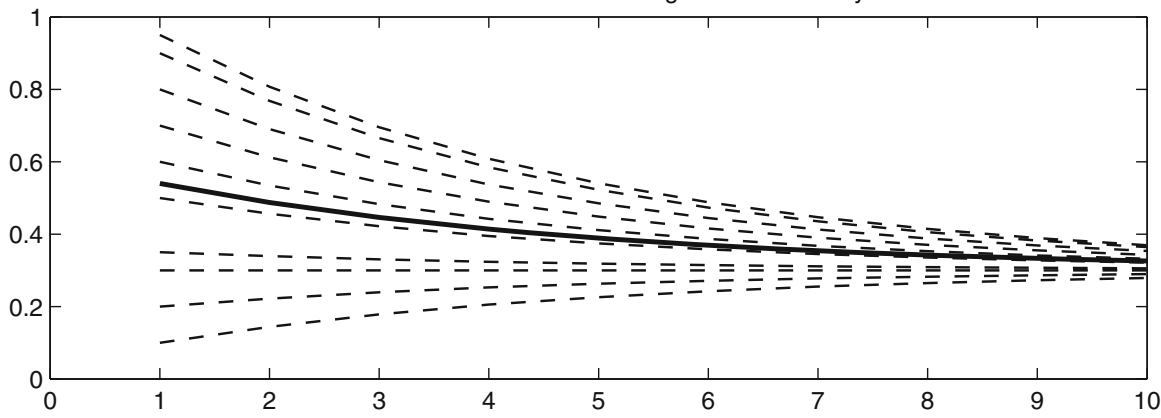

Fig. 1 Individual differences and conformity (Solid Line average salience of individual preferences; Dashed Lines saliences of individual preferences) 
next section, I choose a deliberately arbitrary, but nevertheless plausible parameter value of 0.975 for $\beta$. If $\kappa=40$, there is no systematic deviation between the salience of the private initial convictions and the salience of the social opinion. Additionally, it is assumed that $\mu=\kappa=40$, which implies that individuals dislike deviations from private habits in the same way that they dislike deviations from conformity. These parameter values imply that there is a monotonic convergence to the stationary equilibrium, because $0<\frac{\kappa \beta}{\mu+\kappa}<1$. The parameter values chosen for the remaining simulation exercises also satisfy this condition.

\section{Understanding the basic properties of the model}

\subsection{Baseline simulations}

This section presents simulations using the simple model and the parametrization described in the last section to illustrate how the degrees of individuality and conformity impact on the evolution of individual preferences and the difference between individual and social opinions. Table 1 presents illustrative and purely arbitrary initial values $Z_{0}^{p}$ for the individual salience of preferences for 10
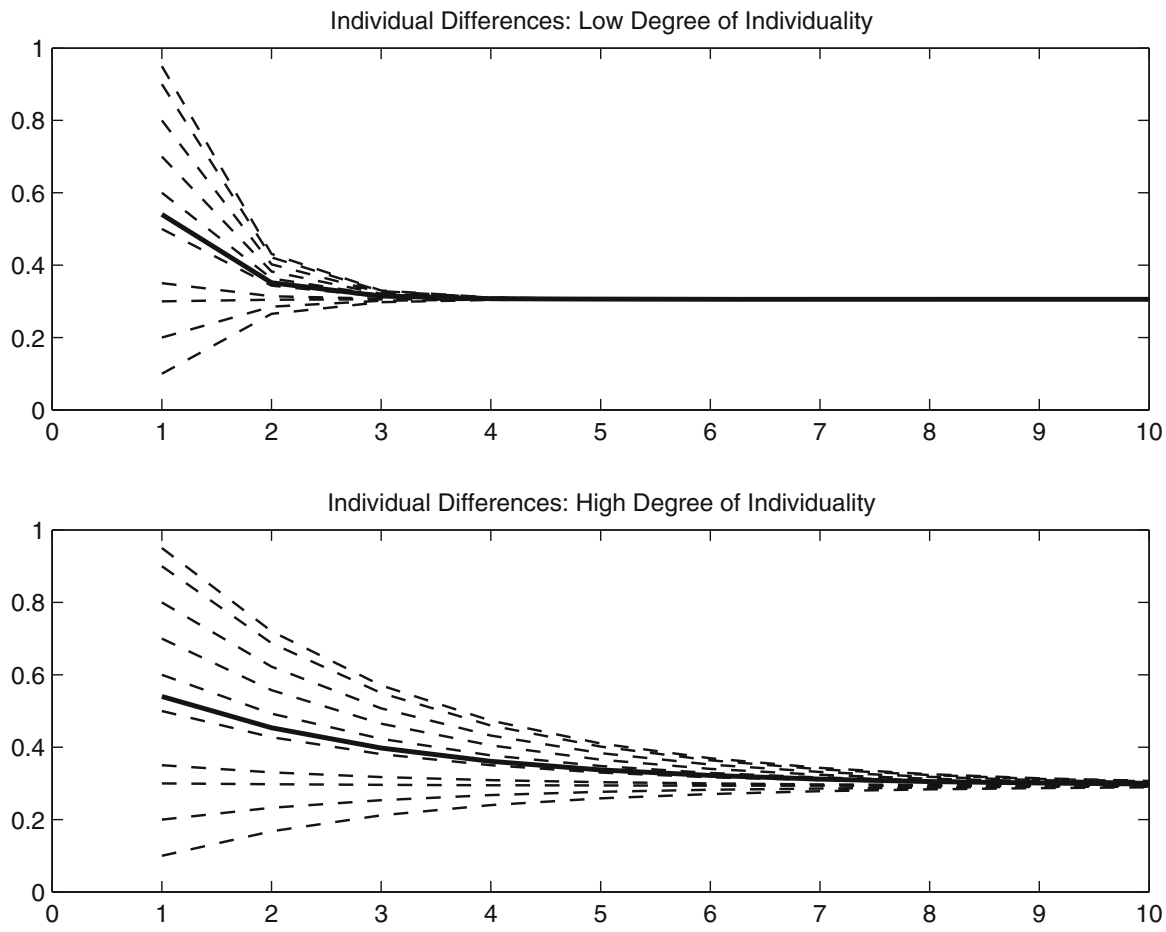

Fig. 2 Individual differences and individuality (Solid Line average salience of individual preferences; Dashed Lines saliences of individual preferences) 

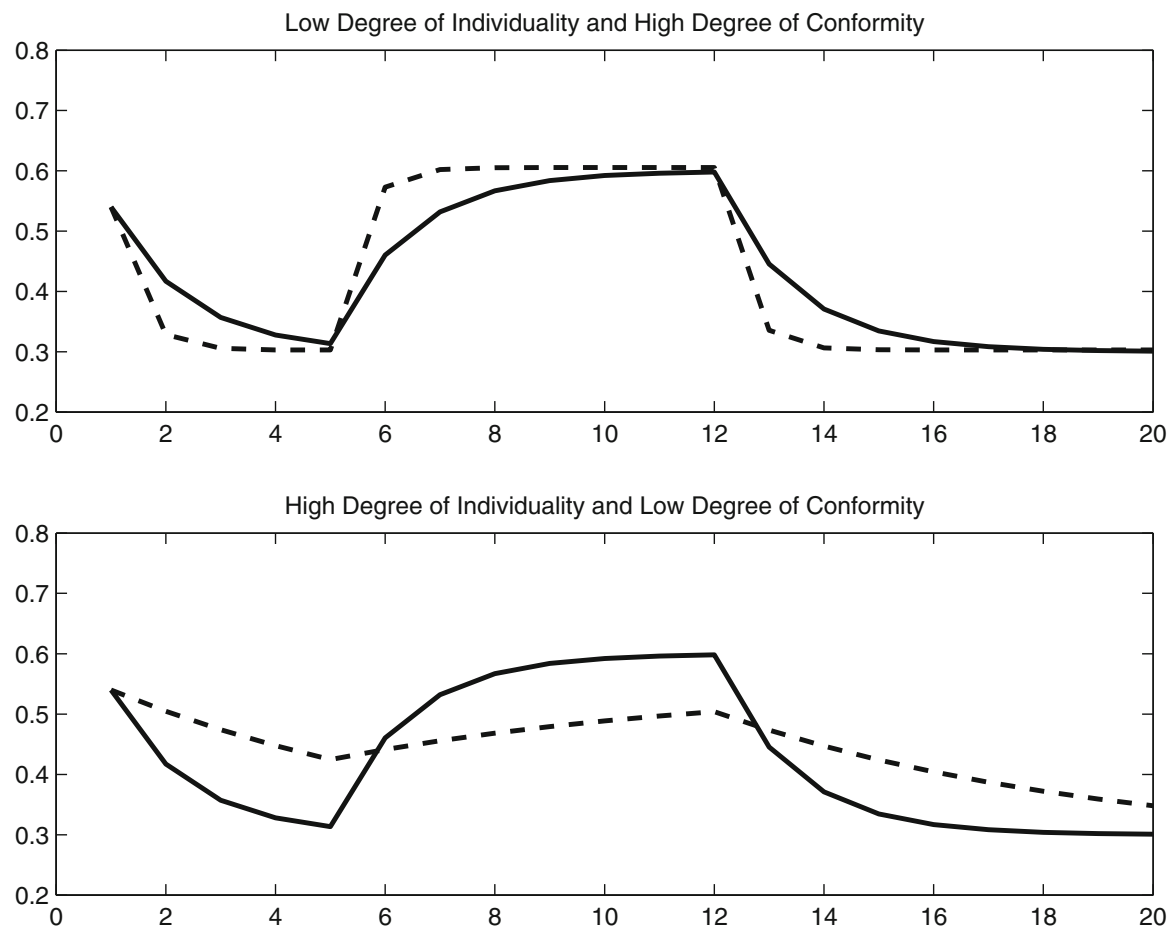

Fig. 3 Long-lasting changes in social opinions (Solid line baseline parameter values; Dashed line Alternative)

individuals. The salience of the social opinion is also chosen in an illustrative and arbitrary way at $Z_{0}^{s}=0.3$. In the baseline version of the model in this subsection, $Z_{t}^{s}$ is held constant at the initial value.

Simple simulation exercises illustrate that individual differences vanish over time, but the pattern depends on the degrees of individuality and conformity. A low degree of individuality $(\kappa=10)$ or a high degree of conformity $(\mu=80)$ reduces the period of time until heterogeneity diminishes, and a high degree of individuality $(\kappa=80)$ or a low degree of conformity $(\mu=10)$ increases this time span (Figs. 1, 2).

\subsection{Temporary changes in social opinions}

This section investigates the effects of when social opinions signal a temporary higher social salience of a preference. Experts, journalists or policy-makers may publicly express changing salience's of preferences for a short period of time. How do conformity and individuality impact on the degree of salience? This is examined by comparing the effects of long- and short-lasting changes in social opinions on the average salience of preferences. Figure 3 analyzes the case where the degrees of individuality and conformity are either lower or higher than in the baseline scenario ( $\kappa=10$ for a low degree and $\kappa=80$ for a high degree of individuality; $\mu=10$ for 


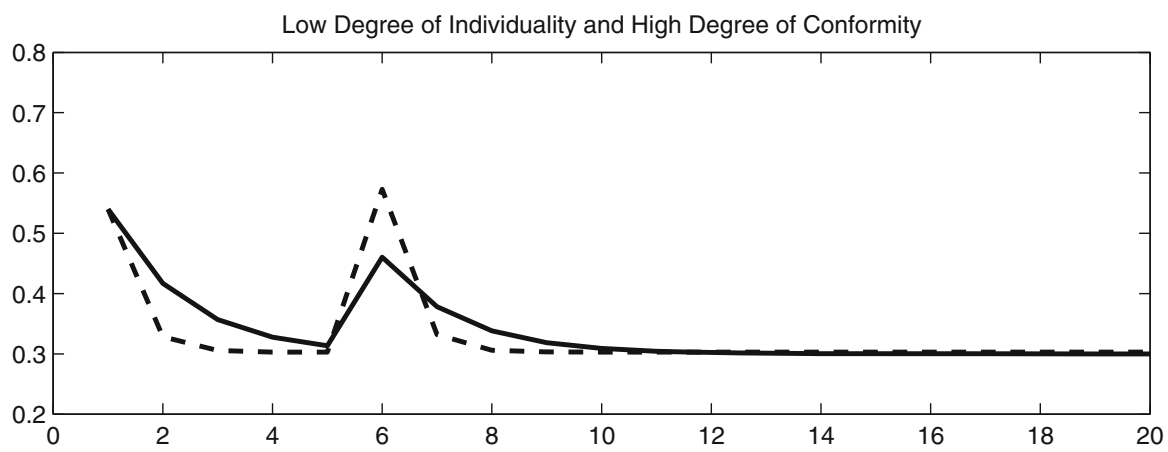

High Degree of Individuality and Low Degree of Conformity

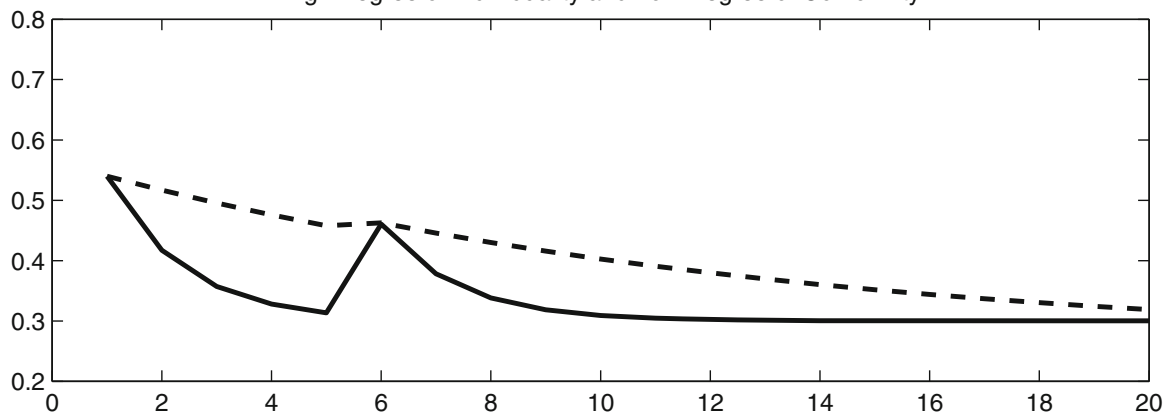

Fig. 4 Short-lasting changes in social opinions (Solid line baseline parameter values; Dashed line Alternative)

a low degree and $\mu=80$ for a high degree of conformity). I assume that from period 6 to 12, social opinions make the salience of a preference temporarily higher than in the rest of the time span (reflected by a temporary increase of $Z_{t}^{s}$ from 0.3 to 0.6), due to modifications in the judgement of the experts, policy-makers, or journalists. One can see that a low degree of individuality and a high degree of conformity lead to a fast reaction to modifications in social opinions. If, however, the degree of individuality is high and conformity is low, individuals do not adapt quickly to temporary changes in social opinions. Figure 4 depicts the case where changes in social opinions only last for one period and this change occurs in period 6. In this case, one could argue that the experts or policy-makers err for a short period of time. A high degree of individuality and a low degree of conformity imply that the temporary judgment errors reflected in the social norms have only a minor impact on the salience of preferences.

This simple analysis shows that societies allowing a high degree of individuality or showing a low degree of social conformity are not subject to overly fast changes if social opinions are only altered for a very short period due to a short-term failure of judgement by experts or politicians. However, at the same time, high individuality and low conformity result in delayed adjustments when new circumstances change social opinions for a long-period of time. 


\section{Conclusion}

This paper has presented a simple model to illustrate how individuality and conformity influence the salience of preferences of individuals. As I argued in this paper, endogenous preference formation may be particularly important in macroeconomic contexts that are often characterized by a high degree of abstractness, uniqueness and uncertainty. Using a simple model, I illustrated how the salience of preferences changes with different degrees of individuality and conformity. Simple simulation exercises show that a high degree of individuality or a low degree of conformity can lead to delayed adjustments, while a low degree of individuality or a high degree of conformity can lead to overreactions and inappropriate choices when social opinion makers err for a short period of time. Individuality and conformity, which are basic and crucial features of how a society is organized, play important roles in the decision-making processes and have a significant influence on the relative salience of preferences. The approach presented in this paper could be useful in implementing simple processes in endogenous preference formation. However, this paper is purely theoretical and does not include empirical applications.

Acknowledgments I would like to thank two anonymous referees and the editors of this journal for very useful comments.

\section{References}

Bernheim BD (1994) A theory of conformity. J Polit Econ 102:841-877

Bowles S (1998) Endogenous preferences: the cultural consequences of markets and other economic institutions. J Econ Lit 36:75-111

Bowles S (2004) Microeconomics: behavior, institutions, and evolution. Princeton University Press, Princeton

Dietrich F, List C (2013) A reason-based theory of rational choice. Noûs 47(1):104-134

Elster J (2009) Reason and rationality. Princeton University Press, Princeton

Harstad RM, Selten R (2013) Bounded-rationality models: tasks to become intellectually competitive. J Econ Lit 51:496-511

Hodgson GM (2004) Reclaiming habit for institutional economics. J Econ Psychol 25:651-660

Hoff K, Stiglitz JE (2010) Equilibrium fictions: a cognitive approach to societal rigidity. Am Econ Rev 100:141-146

Knack S, Keefer P (1997) Does social capital have an economic payoff? A cross-country investigation. Q J Econ 112:1251-1288

Rabin M (1994) Cognitive dissonance and social change. J Econ Behav Organ 23(5):177-194

Rabin M (2013) Incorporating limited rationality into economics. J Econ Lit 51(2):528-543

Ravn M, Schmitt-Grohe S, Uribe M (2006) Deep habits. Rev Econ Stud 73(1):195-218

Rodrik D (2014) When ideas trump interests: preferences, worldviews, and policy innovations. J Econ Perspect 28(1):189-208

Rutherford M (1994) Institutions in economics. Cambridge University Press, Cambridge

Simon HA (1976) From substantive to procedural rationality, method and appraisal in economics. Cambridge University Press, Cambridge 